Supporting Information

\title{
Engineering Magnetosomes for Ferroptosis/Immunomodulation Synergism in Cancer
}

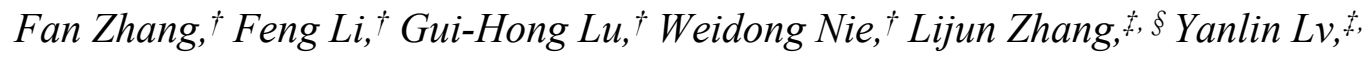

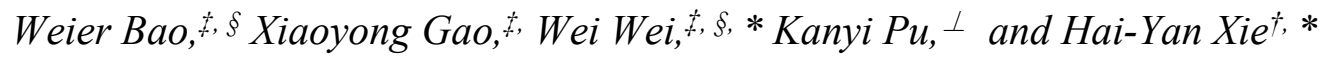

\section{Figures}

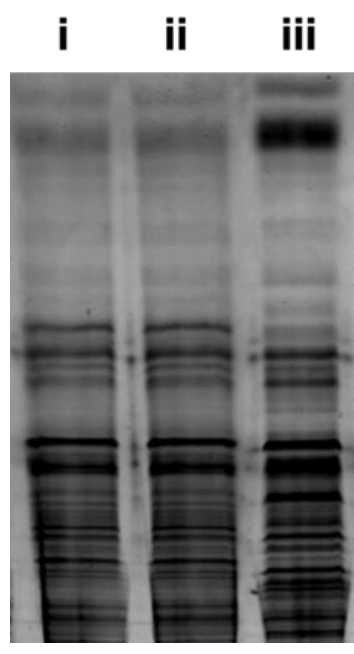

Figure S1. Sodium dodecyl sulfate polyacrylamide gel electrophoresis (SDS-PAGE) analysis of membrane proteins in (i) M-NCs, (ii) the leukocyte membrane and (iii) the whole cell. All results suggested that NCs was successfully coated with the leukocyte membrane. 
PD-1 antibody (Pa)

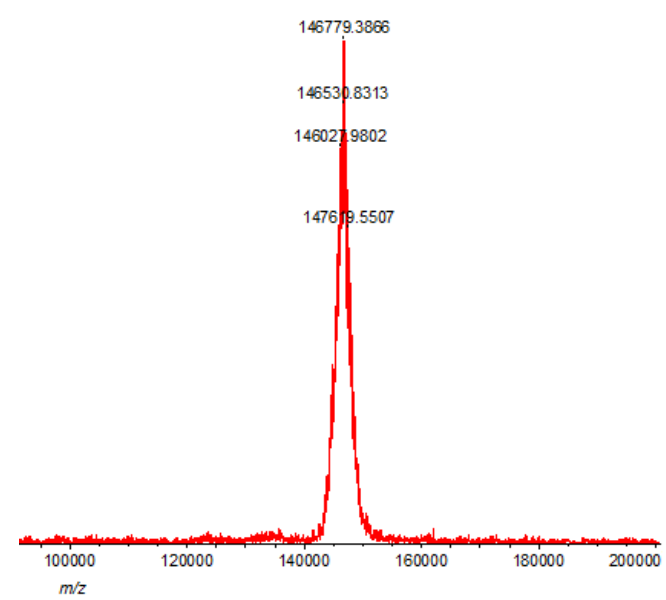

DBCO-Pa

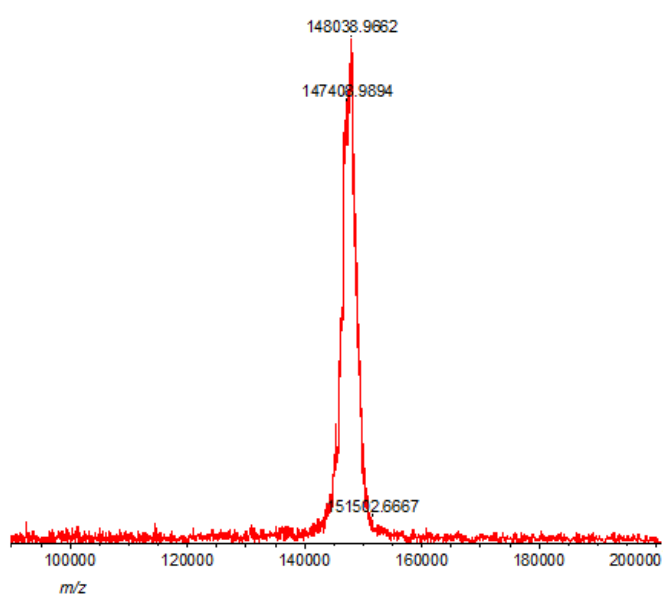

Figure S2. Mass spectra analysis for the DBCO modification of the PD-1 antibody. The relative molecular weight increased by $\sim 1259$ Daltons, which corresponded to 1-2 DBCO.

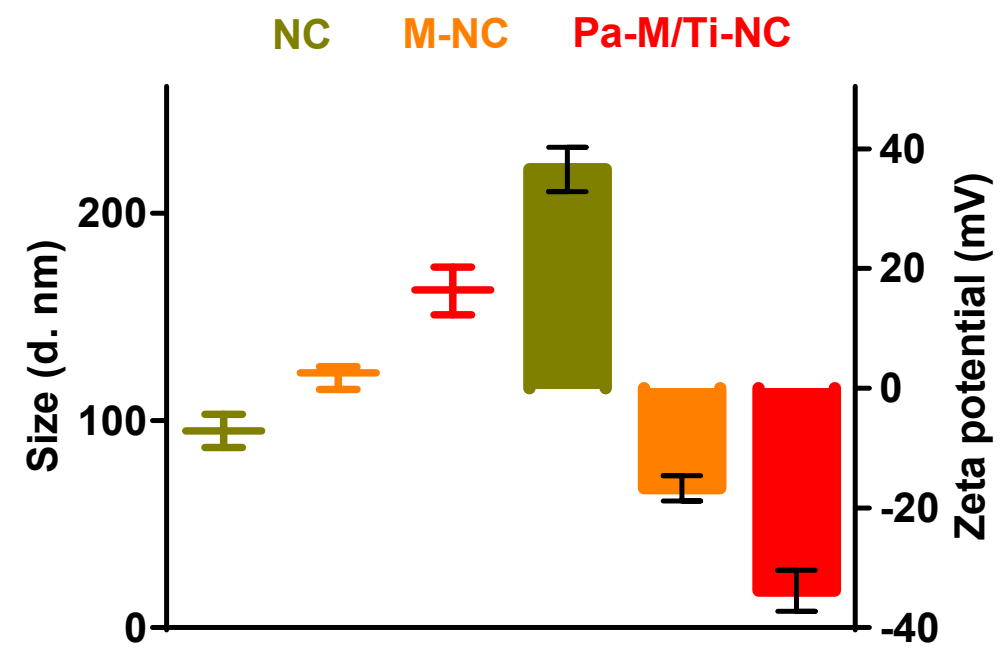

Figure S3. Size and zeta potentials of NCs, M-NCs, and Pa-M/Ti-NCs. Bars represent the mean \pm s.d. $(n=3)$. 

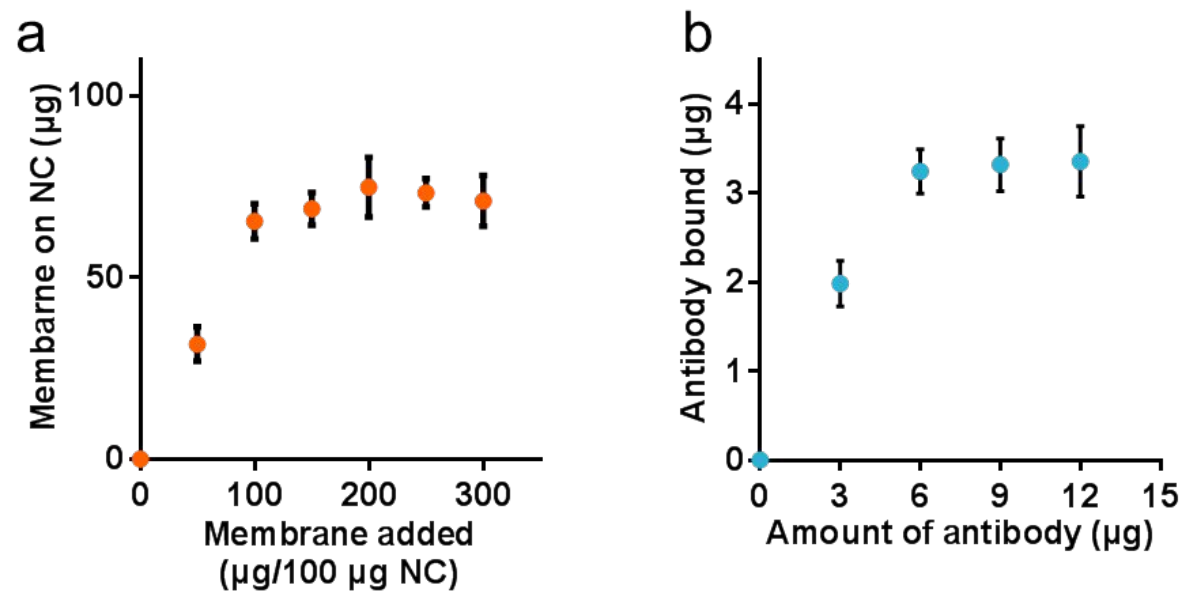

Figure S4. Estimation of the saturation coating of leukocyte membrane fragments on NCs and the saturation of DBCO-Pa on M/Ti-MNCs. a) The coating saturated when $150 \mu \mathrm{g}$ of membrane fragments were used and the amount of membrane attached to the NCs was approximately $70 \mu \mathrm{g}$. b) The total protein amount on Pa-M/Ti-NCs (50 $\mu \mathrm{g}$ ) was measured using a BCA kit after incubation with five serial dilutions of DBCO$\mathrm{Pa}$. According to the data, the decoration became saturated at $\sim 6 \mu \mathrm{g}$ of DBCO-Pa, and the saturated decoration amount was $\sim 3.24 \mu \mathrm{g}$. Bars represent the mean \pm s.d. $(n=3)$.

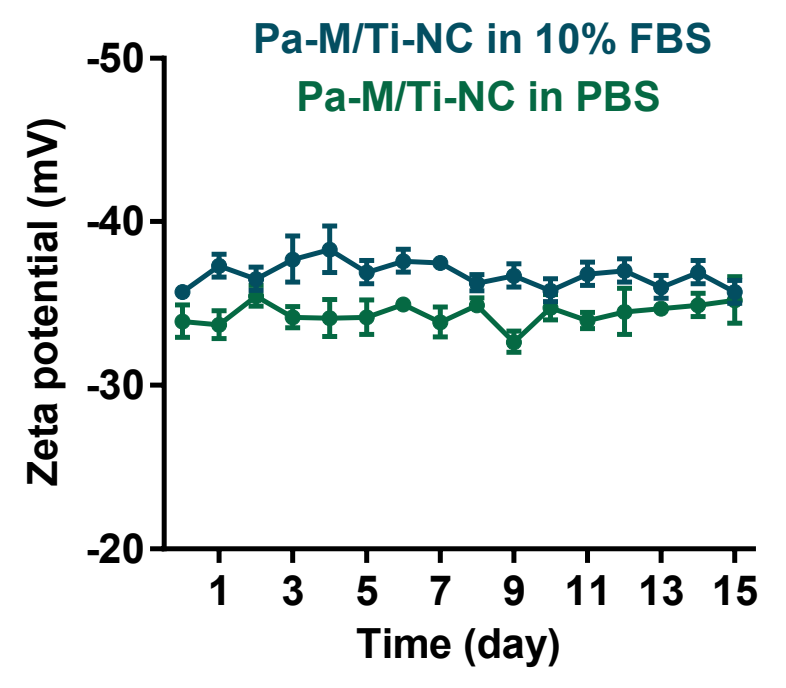


Figure S5. Stability of Pa-M/Ti-NCs. The zeta potentials of Pa-M/Ti-NCs were stable in $10 \%$ FBS and PBS over two weeks, little change was found during storage. Bars represent the mean \pm s.d. $(n=3)$.
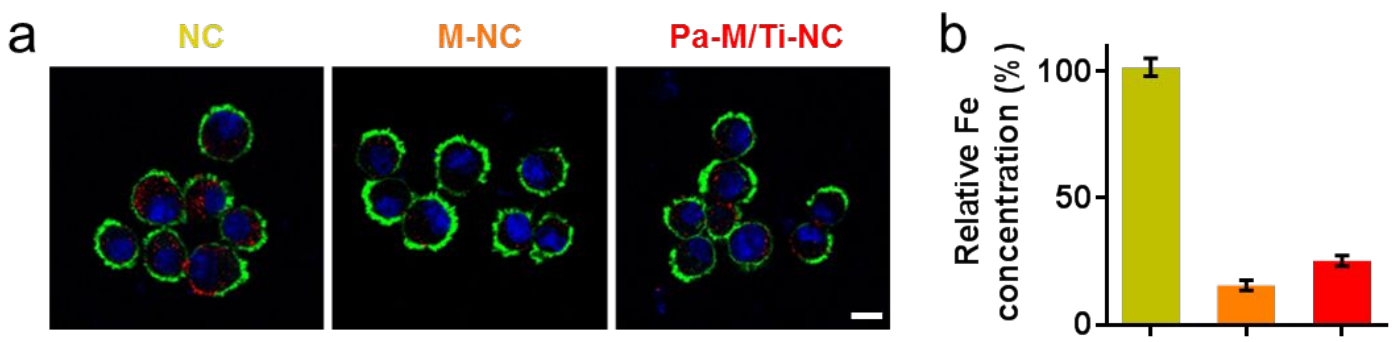

Figure S6. In vitro evaluation of stealth ability and cellular uptake. a) CLSM images of J774A.1 cells after treating with NC, M-NC and Pa-M/Ti-NC (NC-based formulations: red; cytoplasm membrane: green; nucleus: blue, scale bar: $10 \mu \mathrm{m}$ ) and b) relative intracellular Fe concentrations. Bars represent the mean \pm s.d. $(n=3)$.

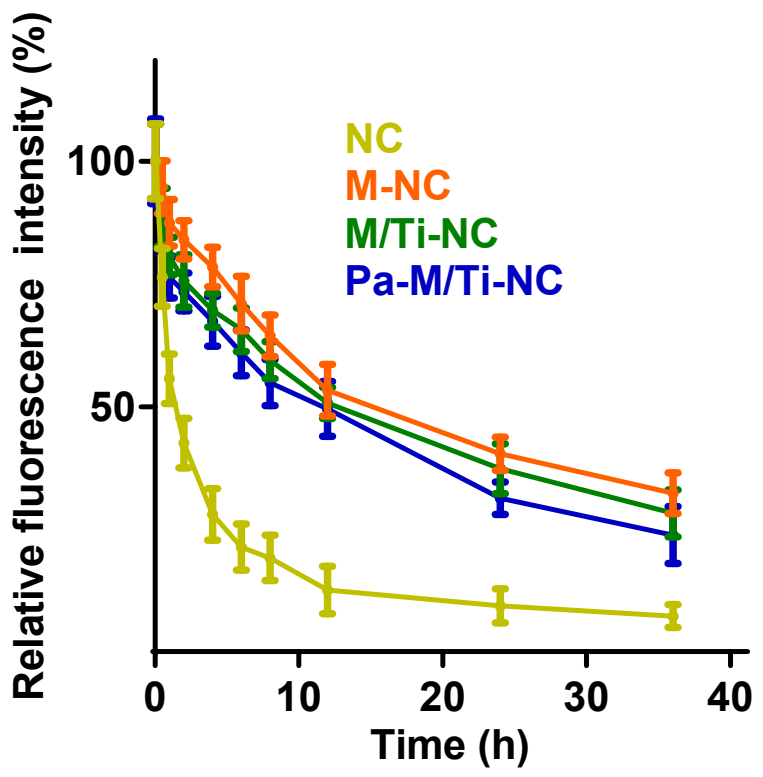

Figure S7. In vivo pharmacokinetic curves over a span of $36 \mathrm{~h}$ after the injection of different formulations (dyed with FITC). At various time points after the injection, blood was collected for the MFI determination. The leukocyte membrane helped NC- 
based formulations enhance blood retention over a span of $36 \mathrm{~h}$. Bars represent the mean \pm s.d. $(n=6)$.

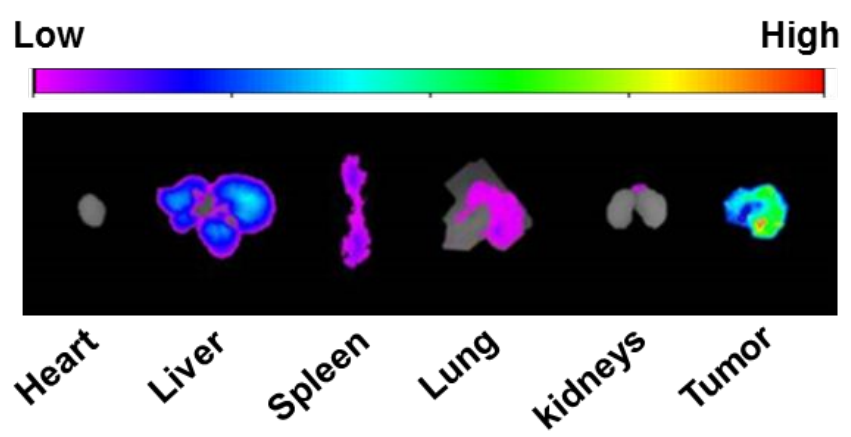

Figure S8. Images of excised tumors and visceral organs at $24 \mathrm{~h}$ after i.v. injection of Pa-M/Ti-NCs (m) labeled with DIR dye. Images are representative of six independent experiments (B16F10 model).

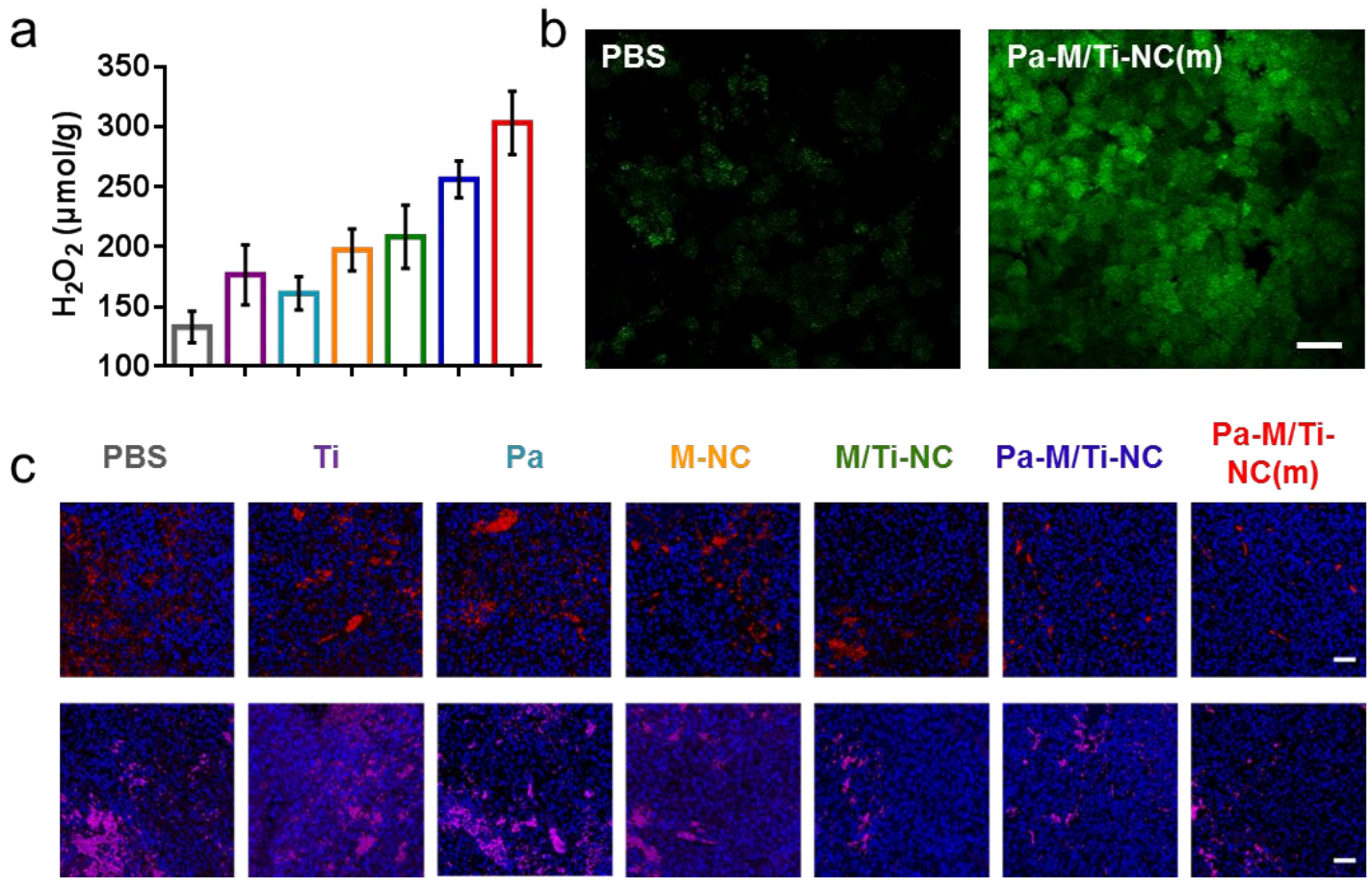

Figure S9. The Pa-M/Ti-NC(m)-induced tumor immune microenvironment changes and ferroptosis of 4T1 tumor model. a) The production of $\mathrm{H}_{2} \mathrm{O}_{2}$ in the tumor 
tissues of different groups. b) CLSM images of hydroxyl radical generation (green) in tumor tissues (scale bar: $10 \mu \mathrm{m}$ ). Together with the released Fe ions from the NC core, an abundance of radicals could thus be generated via the Fenton reaction. c) Immunohistochemical analyses of FACL4 (red) and GPX4 (purple) in tumor tissues (Scale bars: $100 \mu \mathrm{m}$ ).

PBS Ti Pa M-NC M/Ti-NC Pa-M/Ti-NC Pa-M/Ti-NC(m)

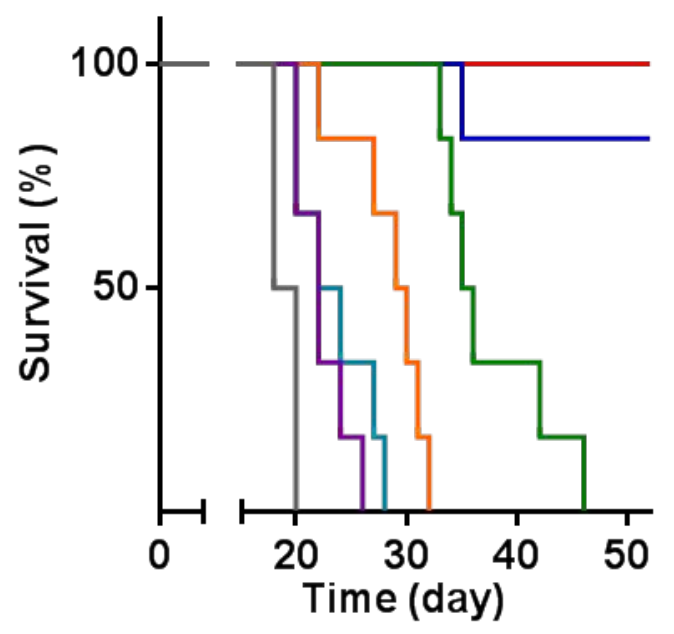

Figure S10. Pa-M/Ti-NCs(m)-mediated tumor inhibition in B16F10 model. The survival of mice showed the antitumor efficacies of the mice receiving different treatments.

PBS Ti Pa M-NC M/Ti-NC Pa-M/Ti-NC Pa-M/Ti-NC(m)
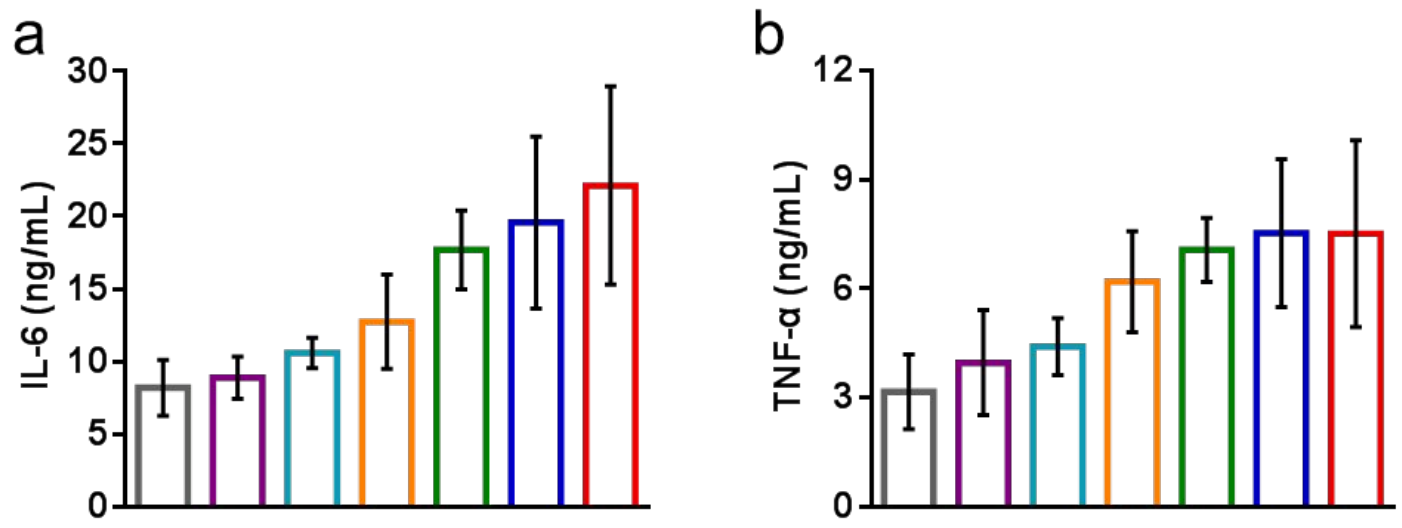
Figure S11. Amount of IL-6 and TNF- $\alpha$ released from mice receiving different treatments with different NC-based formulations. The concentrations of both cytokines were within the normal range in all groups. Bars represent the mean \pm s.d. $(n=6)$.

PBS Ti Pa M-NC M/Ti-NC Pa-M/Ti-NC Pa-M/Ti-NC(m)
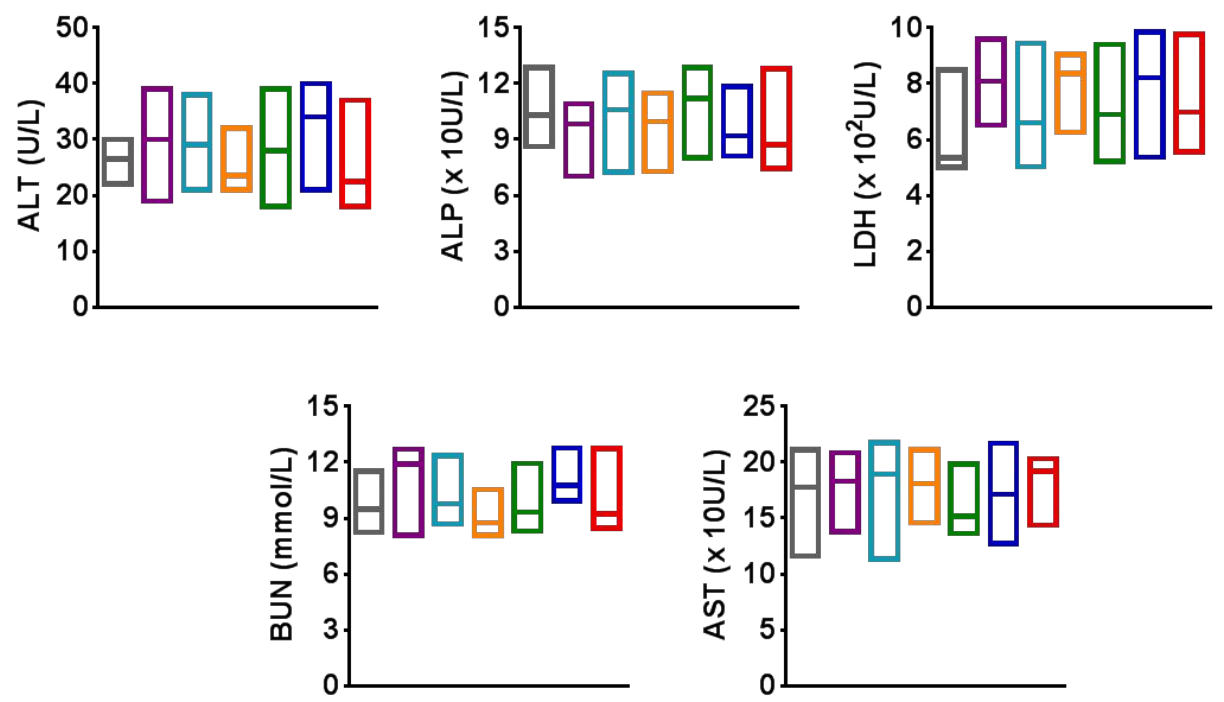

Figure S12. Side effect evaluation via serum biochemical parameter detection. The aspartate alanine aminotransferase (ALT), alkaline phosphatase (ALP), lactate dehydrogenase (LDH), blood urea nitrogen (BUN) and aminotransferase (AST) levels. All the markers were within normal ranges. Bars represent the mean \pm s.d. $(n=6)$.

$\begin{array}{llllll}\text { PBS Ti } & \mathrm{Pa} & \mathrm{M}-\mathrm{NC} & \mathrm{M} / \mathrm{Ti}-\mathrm{NC} & \mathrm{Pa}-\mathrm{M} / \mathrm{Ti}-\mathrm{NC} & \mathrm{Pa}-\mathrm{M} / \mathrm{Ti}-\mathrm{NC}(\mathrm{m})\end{array}$
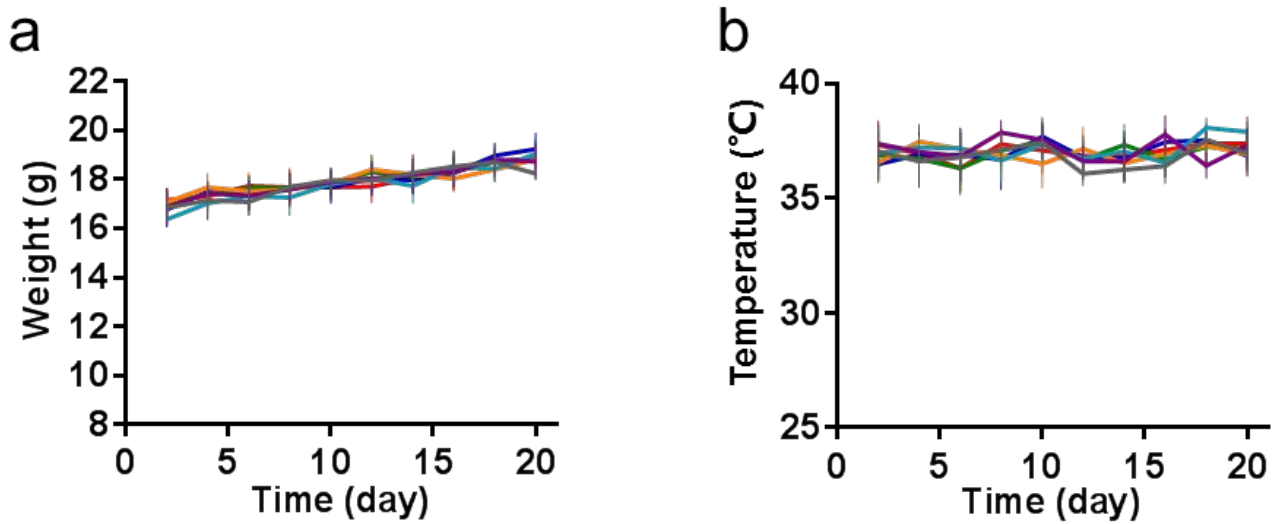
Figure S13. Body weight and temperature variations of B16F10-bearing mice received treatment with different $\mathrm{NC}$-based formulations. There was almost no changes in all groups. Bars represent the mean \pm s.d. $(n=6)$.

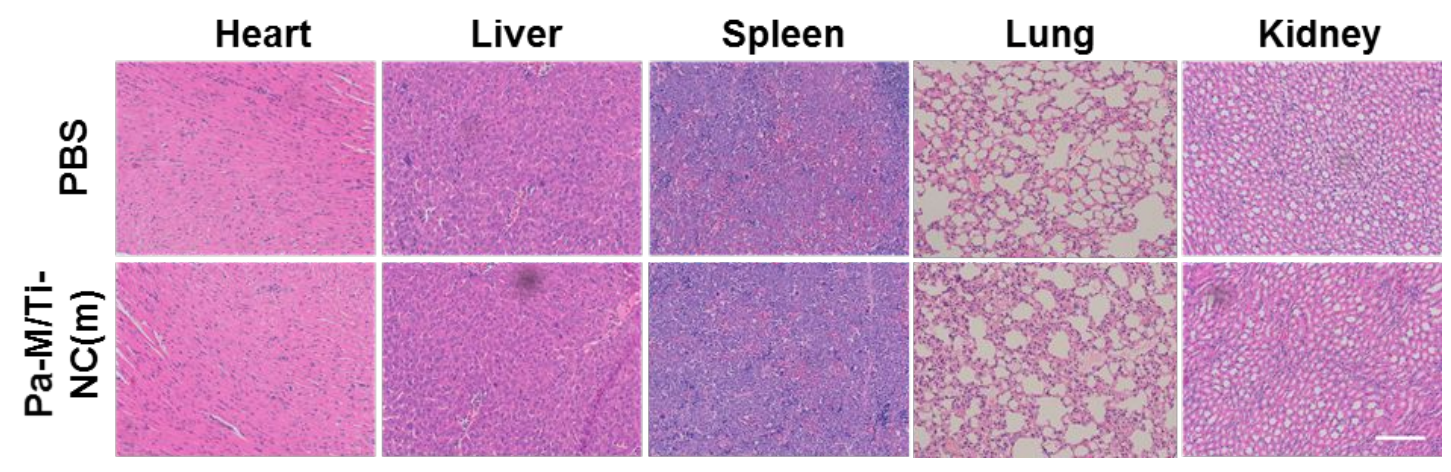

Figure S14. Hematoxylin and eosin (HE) staining of B16F10-bearing mice organs after treatment with Pa-M/Ti-NCs(m) (7 days after administration). No organic damage was observed. Scale bar: $100 \mu \mathrm{m}$.

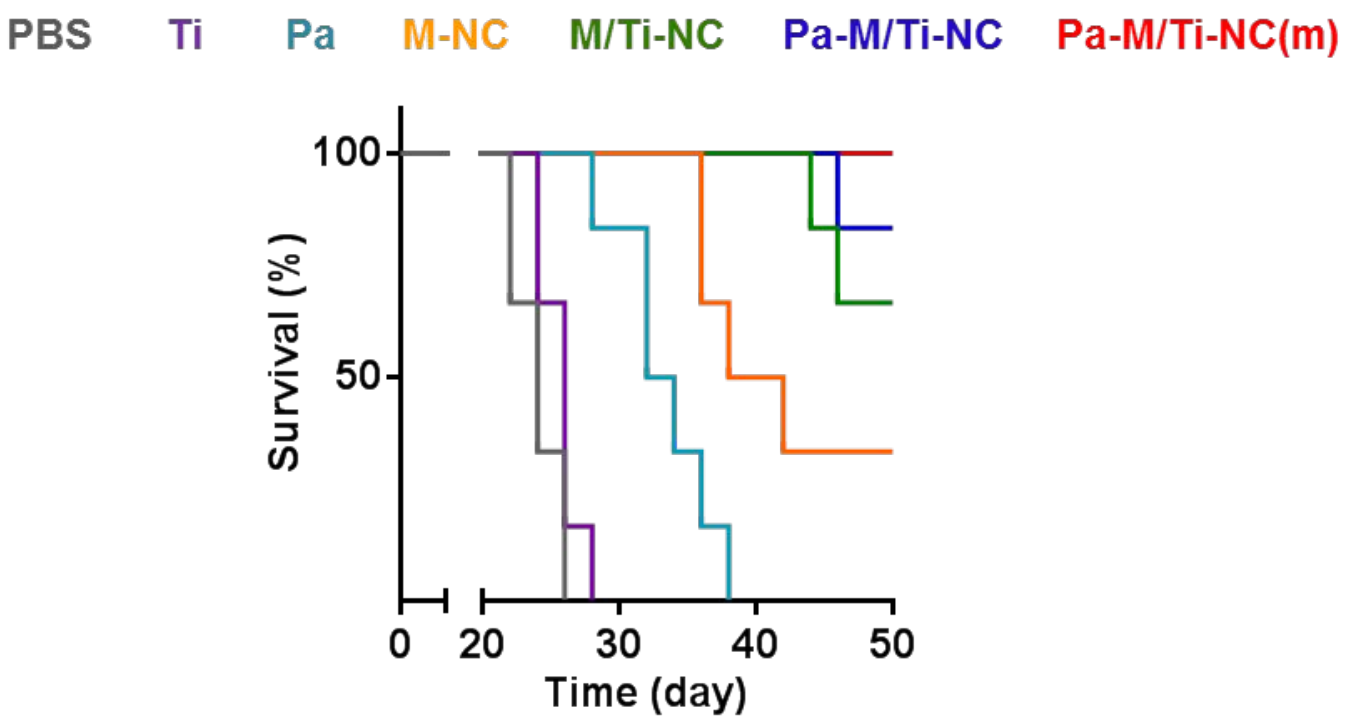

Figure S15. The survival of the mice in 4T1 model. The Pa-M/Ti-NC(m) treatment led to a $100 \%$ survival rate after $50 \mathrm{~d}$. 
PBS Ti Pa M-NC M/Ti-NC Pa-M/Ti-NC Pa-M/Ti-NC(m)
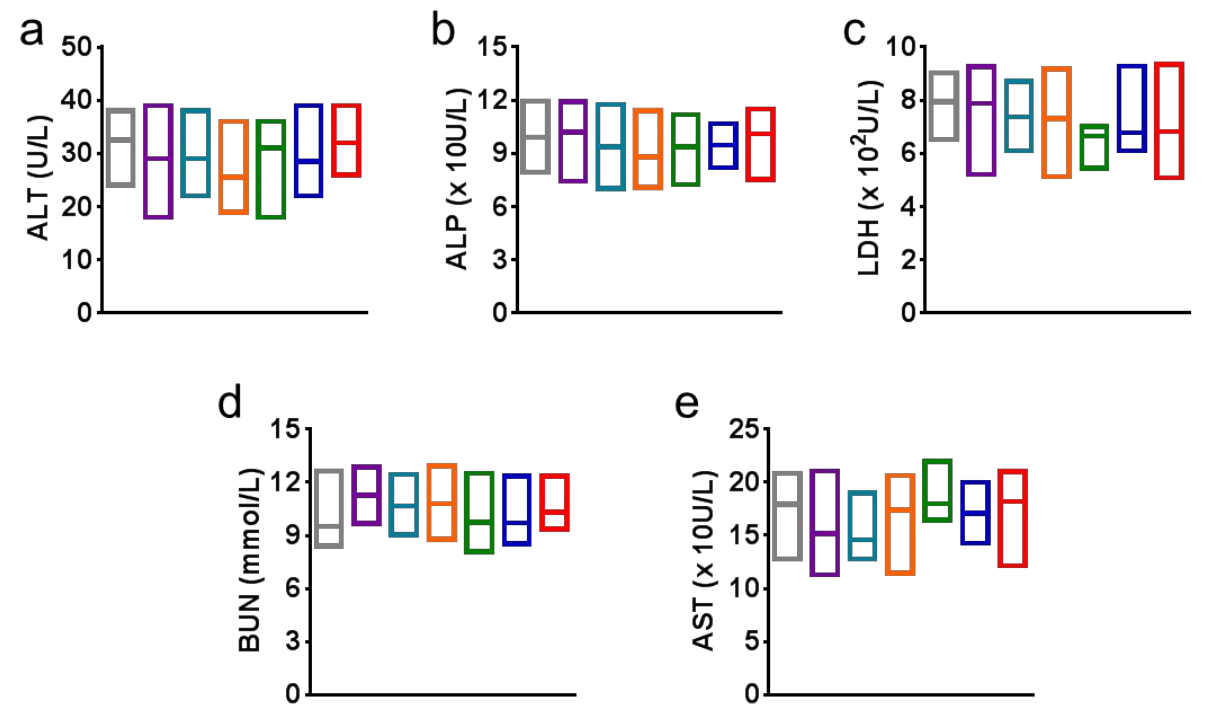

Figure S16. Side effect evaluation via serum biochemical parameter detection of 4T1 tumor model. The aspartate alanine aminotransferase (ALT), alkaline phosphatase (ALP), lactate dehydrogenase (LDH), blood urea nitrogen (BUN) and aminotransferase (AST) levels. All the markers were within normal ranges. Bars represent the mean \pm s.d. $(\mathrm{n}=6)$.

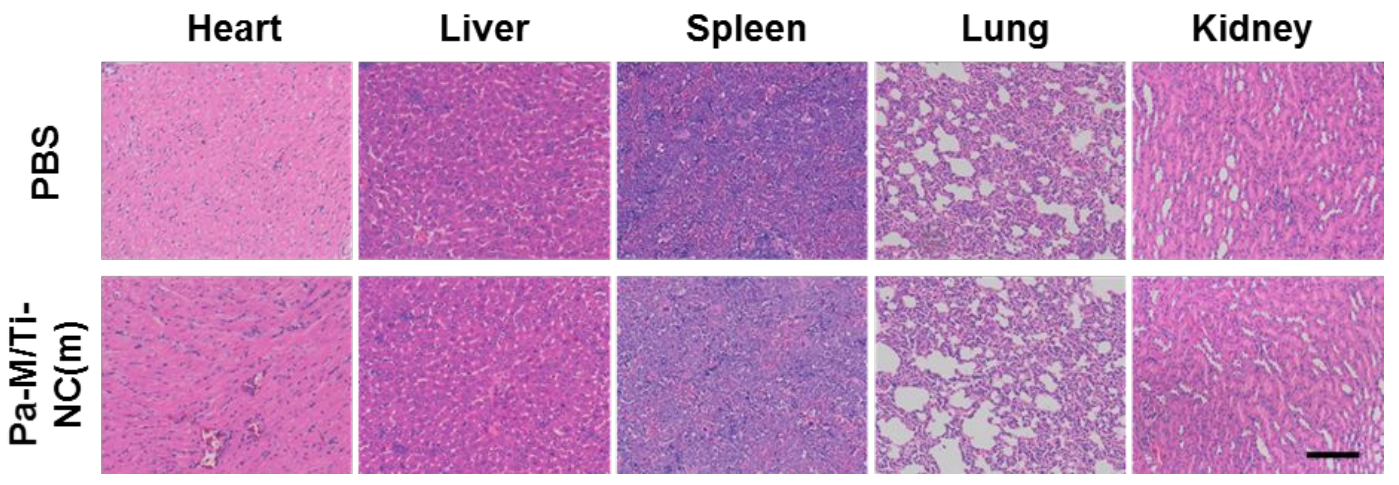


Figure S17. Hematoxylin and eosin (HE) staining of 4T1-bearing mice organs after treatment with $\mathrm{Pa}-\mathrm{M} / \mathrm{Ti}-\mathrm{NC}(\mathrm{m})$ (7 days after administration). No organic damage was observed. Scale bar: $100 \mu \mathrm{m}$.

i: PBS ii: Ti iii: Pa iv: M-NC v: M/Ti-NC vi:Pa-M/Ti-NC vii: Pa-M/Ti-NC(m)

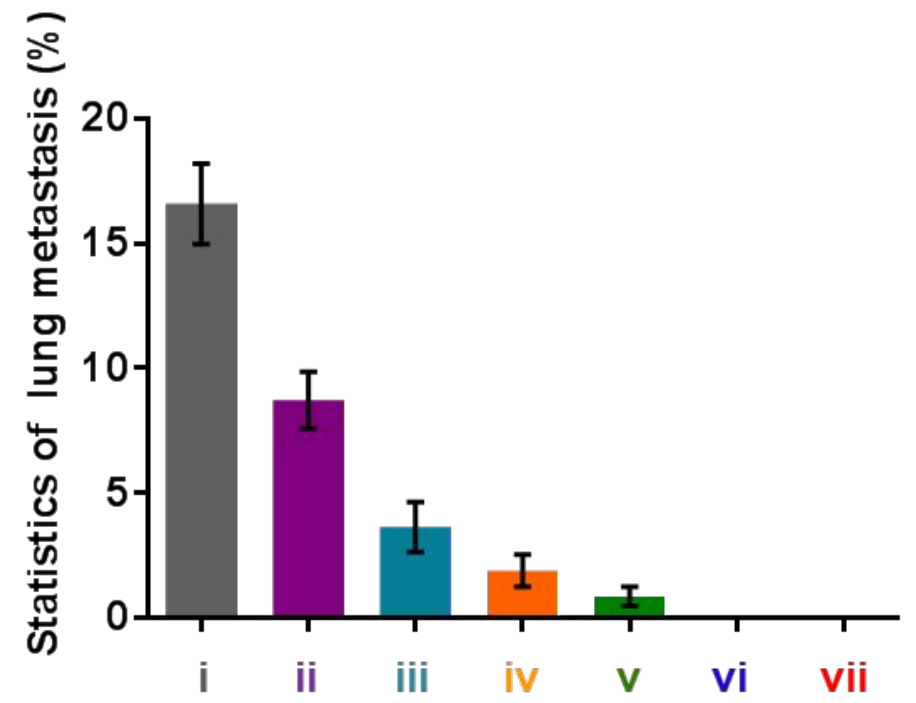

Figure S18. The tumors lung metastasis statistics of 4T1 tumor model. The volume of metastatic sites in lungs decreased in the order of PBS, Ti, Pa, M-NC, M/Ti-NC, PaM/Ti-NC and Pa-M/Ti-NC(m). All data represent the mean \pm s.d. $(n=6)$

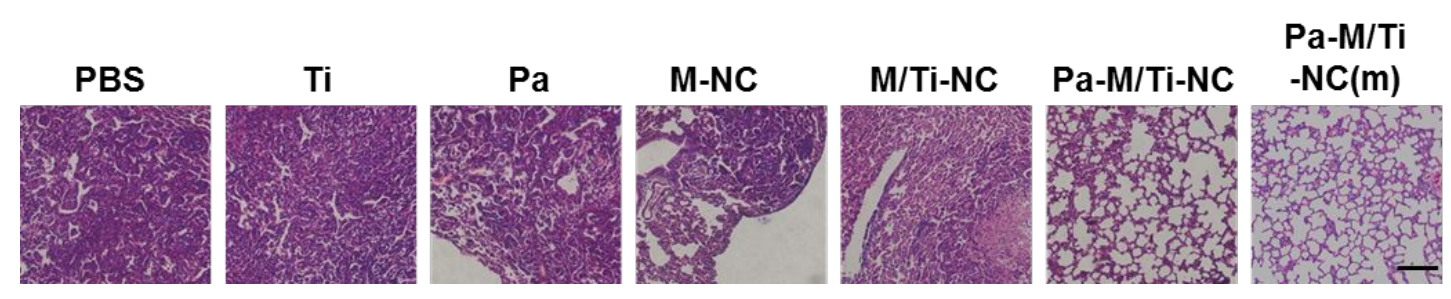

Figure S19. H\&E stained lung sections of 4T1-bearing mice after treatment with different groups. Scale bar: $100 \mu \mathrm{m}$. 
a

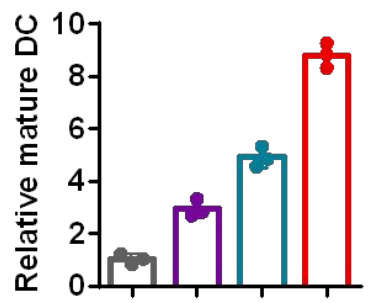

b

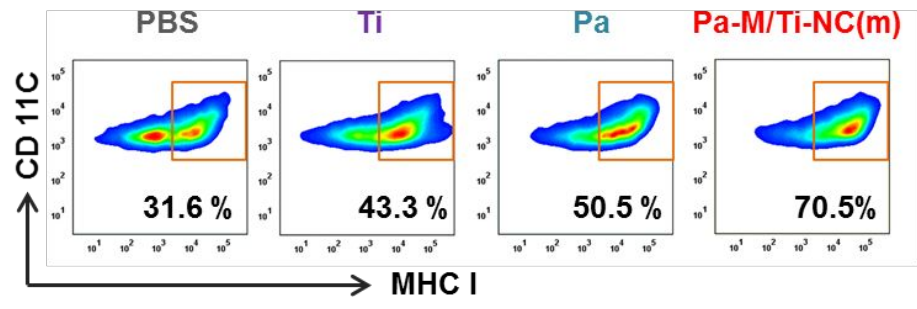

Figure S20. Pa-M/Ti-NC(m)-induced long-term immune memory effects of 4T1 tumor model. a) Relative mature DC in primary tumors with different treatment on the resection day. b) Flow cytometry analysis of DC cross-presentation marker MHC-I in a. All data represent the mean \pm s.d. $(n=6)$.

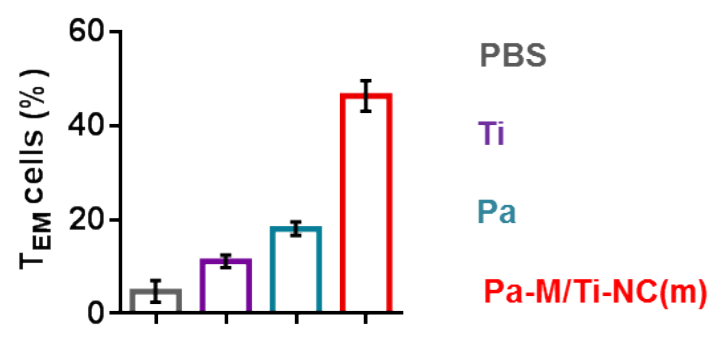

Figure S21. Percent of $T_{E M}$ cells in spleens of the mice on the same day of rechallenging (4T1 tumor mode). All data represent the mean \pm s.d. $(n=6)$. 